\title{
Inflammatory ascending aortic disease: Perspectives from pathology
}

\author{
Joseph J. Maleszewski, MD
}

\begin{abstract}
Inflammatory diseases of the aorta comprise a spectrum of disease with diverse clinical and histopathologic presentations. Broadly, they may be dichotomized into infectious and noninfectious varieties. Although uncommon, infectious forms, caused by bacteria, fungi, or mycobacteria, may result from hematogenous seeding of the aorta or direct spread from a contiguous infectious source. The noninfectious forms include a number of entities, the most common of which is atherosclerosis, a disease that primarily affects the aortic intima but has important secondary effects on the media and adventitia that may result in aneurysm formation. Other important noninfectious inflammatory diseases include giant cell arteritis, Takayasu arteritis, granulomatosis with polyangiitis (Wegener granulomatosis), sarcoidosis, and lymphoplasmacytic aortitis. Importantly, there is increasing recognition that there is a subset of cases of lymphoplasmacytic aortitis perhaps better classified under the spectrum of so-called IgG4-related sclerosing disease, with important clinical and therapeutic ramifications. This review focuses on the variable and defining characteristics of the inflammatory aortopathies, specifically those affecting the ascending aorta, and discusses areas of important clinical and pathological distinction between them. (J Thorac Cardiovasc Surg 2015;149:S176-83)
\end{abstract}

\section{AORTIC ANATOMY}

The aorta is the largest elastic artery in the body. Elastic arteries are defined by the presence of concentrically arranged lamellar units forming their media. A lamellar unit consists of collagen fibers, smooth muscle myocytes, and ground substance sandwiched between the concentric elastic lamella (plates). ${ }^{1}$ The elastic lamellae are anchored to one another by smaller elastic fibers. ${ }^{2}$

There are important structural differences between the thoracic and abdominal aortic regions. ${ }^{3}$ In fact, there are even structural differences that have been noted between different regions of the ascending aorta, specifically the sinus and tubular portions. ${ }^{4}$ These differences are likely related to the different embryologic origins of the aorta, with the root being derived primarily from second heart field (lateral plate mesoderm), the ascending aorta and arch from the neural crest, and the descending aorta from paraxial mesoderm (Figure 1). ${ }^{5}$ This regional heterogeneity in aortic structure is undoubtedly an important determinant of how the aorta responds to injury or degeneration.

\section{SPECIMEN HANDLING}

Surgical ascending aortic specimens are received in the pathology laboratory in 1 of 2 varieties: an unopened segmental resection (Figure 2, A) including a full-

From the Division of Anatomic Pathology, Mayo Clinic, Rochester, Minn.

Disclosures: Author has nothing to disclose with regard to commercial support.

Read at The American Association for Thoracic Surgery Aortic Symposium, New York, New York, April 24-25, 2014.

Received for publication May 21, 2014; accepted for publication July 24, 2014; available ahead of print Sept 5, 2014.

Address for reprints: Joseph J. Maleszewski, MD, Mayo Clinic, 200 First St SW, Rochester, MN 55905 (E-mail: maleszewski.joseph@mayo.edu).

$0022-5223 / \$ 36.00$

Copyright (C) 2015 by The American Association for Thoracic Surgery

http://dx.doi.org/10.1016/j.jtcvs.2014.07.046 circumference portion of the aorta, or an opened specimen in which either a full- or partial-circumference portion of aorta has been removed (Figure 2, B). Once it has been received by the pathology laboratory, the specimen is measured, oriented, and described. In the event that the specimen either is noncircumferential or was previously opened, orientation may be facilitated by placement of a marker suture in the sinus portion of the aorta. Such orientation allows for appropriate sampling and evaluation, specifically because of the aforementioned differences in regional histologic properties.

The adventitia, media, and intima are carefully examined and described in the gross pathology report. From 6 to 8 full-thickness sections of aortic wall are then typically procured for paraffin embedding and sectioning (Figure 3). ${ }^{6}$ Tissue sections are then stained with hematoxylin and eosin as well as an elastic stain (eg, Verhoeff-van Gieson stain; Figure 4) for review by the pathologist.

\section{INFLAMMATORY AORTIC DISEASE}

Aortitis is broadly defined as inflammation of the aortic wall. In general, conditions that result in secondary inflammation are not considered aortitis. Atherosclerosis, an inflammatory aortic disease primarily affecting the intima, is also typically not considered aortitis as such. Nevertheless, it is an important inflammatory disease that may occasionally have a very prominent inflammatory component. Although it more commonly affects the descending aorta, the infrarenal component in particular, it may be encountered in the ascending aorta. In the ascending aorta, the sinotubular junction and the minor curvature are more commonly involved. ${ }^{7}$ Branch points such as the coronary ostia and arch vessels are also particularly vulnerable, presumably because of endothelial dysfunction in regions of hemodynamic instability. ${ }^{8}$ 


\section{Abbreviations and Acronyms \\ GCA = giant cell arteritis \\ GPA $=$ granulomatosis with polyangiitis \\ IgG4-RSD = IgG4-related sclerosing disease- associated aortitis \\ TA $=$ Takayasu aortitis}

Grossly, atherosclerosis may manifest along a spectrum from a yellow-white, irregular endothelial lesion known as a streak to large, variegated, and ulcerated plaques. Ulcerated areas may have a sparkling appearance, which results from the reflection of light off of the cholesterol crystals contained within the lesion. Areas of calcification may be present to varying degrees throughout the involved areas. Luminal thrombus may also be seen along regions of the intimal lesion.

Histologically, atherosclerosis is characterized by an admixture of variable degrees of histiocytes (including socalled foam cells), lymphocytes, and usually cholesterol clefts within intimal plaques. The inflammatory infiltrate may become particularly intense (Figure 5, A) in regions of recent plaque disruption, even producing lymphoid follicles. Neutrophilic inflammation may also be seen, causing concern regarding an underlying infectious process.

Although the aforementioned gross and histologic features are rather specific for atherosclerosis, they do not preclude the presence of an underlying disease that may, in fact, be primary. Because endothelial injury is a common phenomenon in many aortic diseases, the development of secondary atherosclerosis is not uncommon.

So-called inflammatory abdominal aortic aneurysm was historically though to be related to severe atherosclerosis; however, the atherosclerotic process is now believed more likely to be a secondary phenomenon arising out of an underlying issue, such as IgG4-related sclerosing disease. ${ }^{9}$

\section{Noninfectious Aortitis}

Noninfectious aortitis includes a wide array of diseases, including giant cell arteritis (GCA) involving the aorta, Takayasu aortitis (TA), granulomatosis with polyangiitis (GPA), lymphoplasmacytic aortitis, Behçet disease, sarcoidosis, Cogan syndrome, and aortitis associated with other rheumatologic diseases (eg, rheumatoid arthritis and systemic lupus erythematosus). A thorough discussion of each of these entities is beyond the scope of this review. Prototypical diseases with important clinical points are therefore the focus in this section.

GCA involving the aorta. GCA (formerly Horton disease), a vasculitis that may involve the aorta, its branches, and smaller muscular vessels, is the most common systemic vasculitis to involve the aorta. ${ }^{10}$ Such aortic involvement in GCA is relatively common, ranging from $10 \%$ to $40 \%$ in most series. ${ }^{11-13}$ It is usually encountered in individuals older than 50 years and affects women 3 to 5 times more commonly than men. There is also an association with polymyalgia rheumatica. ${ }^{14}$ Nonaortic symptoms include headache, jaw claudication, or visual changes. Alternatively, the disease may manifest as an asymptomatic isolated aortic process, usually presenting as an aneurysm. In fact, most cases of aortic GCA appear to be such isolated involvement. ${ }^{15}$

Grossly, the aortic intima frequently exhibits a wrinkled "tree bark" appearance (Figure 3). This character is imparted by a combination of medial damage, tissue edema, and the elastic properties of the vessel itself. Accordingly, this finding may be encountered in virtually any form of aortitis. Secondary atherosclerosis may also be seen as a result of altered hemodynamics and endothelial dysfunction; however, this finding also is not specific for GCA.

Histologically, GCA is characterized by a granulomatous inflammatory infiltrate, involving the media, with a variable number of multinucleated giant cells (Figure 5, B). A lymphoplasmacytic infiltrate may be present as well. The vessel frequently exhibits a "moth-eaten" appearance on elastic stain as a result of the medial injury (Figure 5, C). The inflammation may involve vasa vasora and may even produce regional bandlike infarction of the media (Figure 5, $D) .{ }^{16}$ This is among the mechanisms of formation of socalled laminar medial necrosis. It is important to note, however, that laminar medial necrosis is neither specific for GCA nor an inflammatory process and may be seen in noninflammatory aneurysms as well.

Takayasu aortitis. TA is the prototypical inflammatory aortic disease of the young. Typically presenting in those younger than 50 years, it often manifests in young women (female to male ratio of 8:1) of Asian descent. As with other forms of aortitis, the disease may be asymptomatic or present with catastrophic complications such as stroke. Its obstructive involvement of the arch vessels may result in loss of circulation to the upper extremities, earning the TA its nickname of "pulseless disease."

Aortic specimens from individuals with TA exhibit variable changes, depending on the phase of the disease at the time of resection. Acute phase disease may appear similar to other cases of aortitis, with areas of wall thinning and intimal wrinkling. Chronic or late phase disease may manifest as mural thickening (sometimes striking), which may involve any of the layers of the vessel but most notably the intima and adventitia. The thickening is often circumferential, producing regions of stenosis in the aortic branches.

Histopathologic features are also dependent on the phase of the disease, although the late phase is more commonly encountered. Acutely, the disease may look identical to GCA, and only the age of the patient can be used to reliably distinguish between these disease processes. In the chronic 


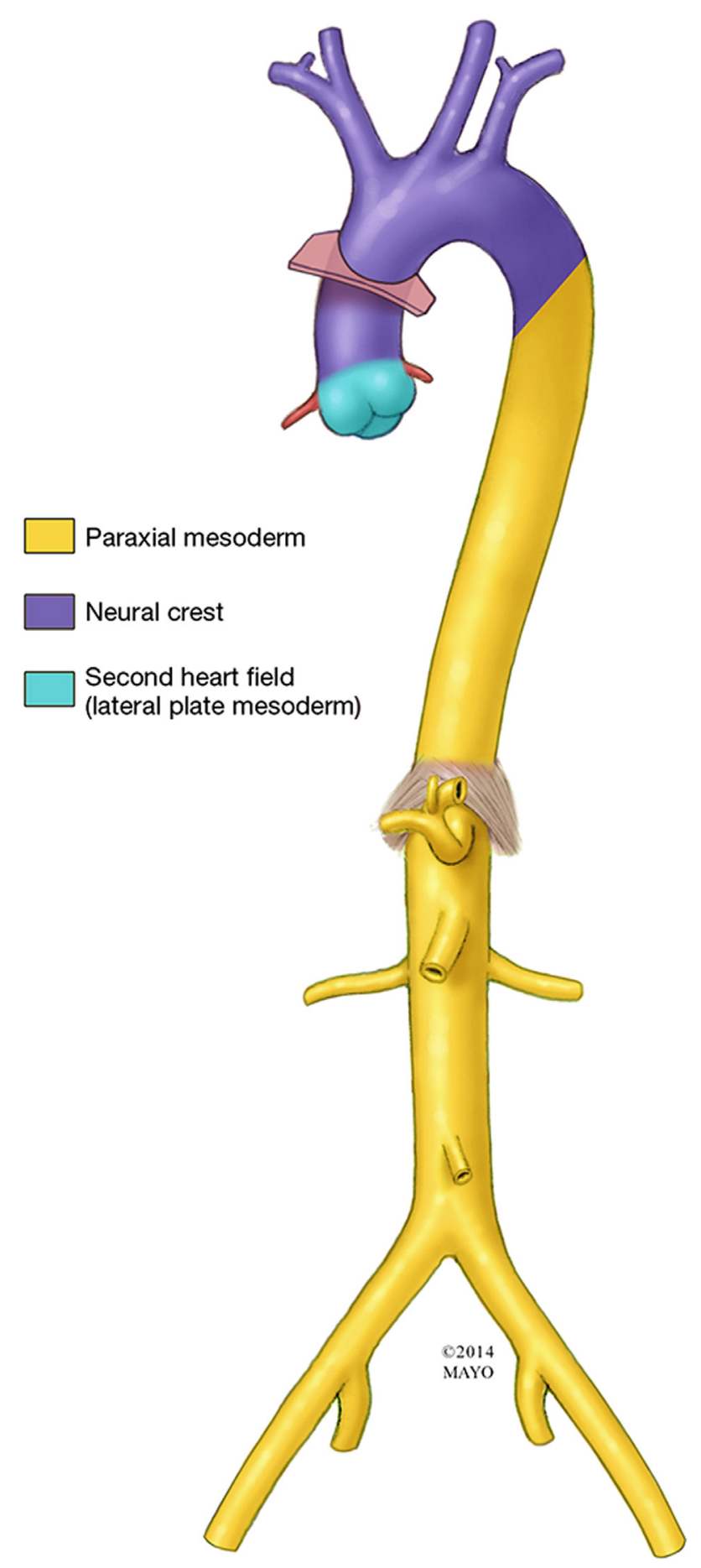

FIGURE 1. Aortic schematic diagram. Regional differences in aortic pathology are probably explained, at least in part, by the varying embryologic origins. The aortic root is derived primarily from the second heart field (lateral plate mesoderm), whereas the ascending aorta and arch are of neural crest derivation. The remaining aorta is derived from the paraxial mesoderm.

phase, there is fibrotic replacement of areas of the media, with fibroplastic and fibrotic thickening of the intima and media, respectively.
Granulomatosis with polyangiitis. GPA (formerly Wegener granulomatosis), is a necrotizing systemic vasculitis that commonly affects the lungs, kidneys, head and neck. In general, presentation occurs between 65 and 70 years of age, with no obvious sex predilection. ${ }^{17,18}$ Although the precise etiology of GPA is unknown, it is clear that there is an autoimmune component of the disease, with the development of antineutrophil cytoplasmic antibodies (ANCA) usually directed against proteinase-3 (so-called c-antineutrophil cytoplasmic antibodies) seen in $60 \%$ to $90 \%$ of cases of GPA. Although such involvement is infrequent, GPA may affect the aorta and its branches. ${ }^{19,20}$

Grossly, GPA manifests much as does other forms of aortitis. Periaortic or adventitial thickening may sometimes be present. Histopathologically, GPA is characterized by dark basophilic areas of necrosis that irregularly interface with the adjacent inflammatory process (so-called geographic necrosis; Figure 5, E). The granulomatous infiltrate often includes darkly staining, multinucleated giant cells. The vasculitis may involve not only the aortic wall but also the vasa vasora.

Sarcoidosis. Sarcoidosis is a systemic disease characterized by the presence of well-formed, nonnecrotizing granulomas. Although its etiology is unknown, cases of familial clustering have been reported. ${ }^{21}$ Typical presentation occurs across a relatively wide age spectrum from 20 to 40 years, although younger presentations are more common among African Americans, a population in which the disease is 3 to 4 -fold more common than among others. Involvement of most organs has been described, including the aorta (albeit rarely). ${ }^{22,23}$ Interestingly, some have also reported cases of a TA-like aortitis in the setting of sarcoidosis, although there is no clear association between these diseases. $^{24,25}$

As previously noted, well-formed, nonnecrotizing granulomatous inflammation is the hallmark of sarcoidosis. This inflammation is often associated with fibrosis. Aortic manifestations may involve any of the wall layers, although adventitial or periadventitial involvement is the most common (Figure 5, F). Concomitant hilar adenopathy, seen at the time of surgery, can be a clue to the diagnosis.

IgG4-related sclerosing disease-associated aortitis. The newly coined but increasingly recognized entity IgG4related sclerosing disease-associated aortitis (IgG4-RSD) most commonly involves the descending aorta, but may involve the ascending aorta as well. IgG4-RSD is characterized by elevated serum levels of $\operatorname{IgG} 4$, fibrosis, and inflammation that contains increased IgG4-positive plasma cells. First recognized as a form of pancreatitis (autoimmune pancreatitis), IgG4-RSD appears to represent a unifying diagnosis among separately described entities such as sclerosing cholangitis, nephritis, sialadenitis (Kuttner tumor), retroperitoneal fibrosis (Ormond disease), and aortitis. 

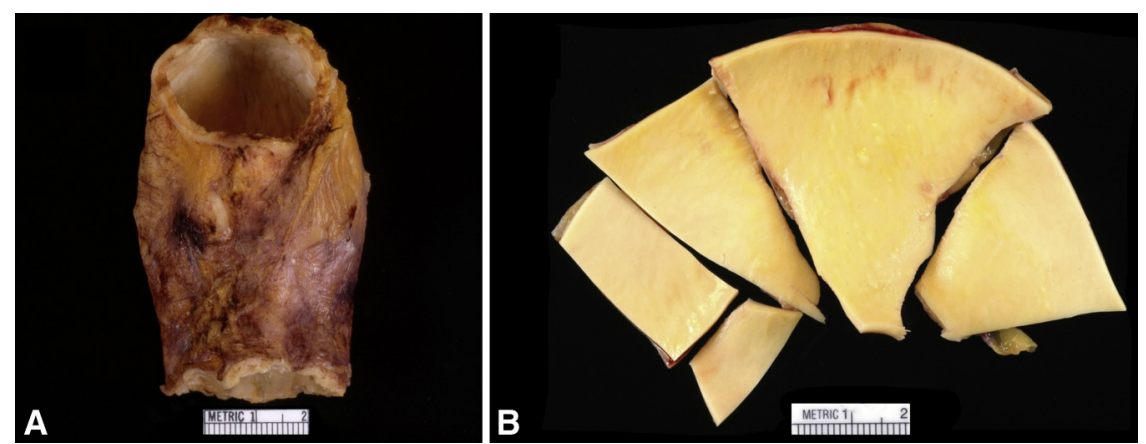

FIGURE 2. Gross specimens. A, This photograph depicts a segmental aortic resection received intact and easily orientable by the pathologist. B, This photograph depicts an opened specimen, difficult to orient and representatively sample.

The disease usually manifests in individuals older than 50 years. Importantly, treatment of IgG4-RSD aortitis is different from that of most other noninfectious forms of aortitis, often consisting of plasmapheresis or anti-B lymphocyte therapy. ${ }^{26}$

Tissue sections of aortic specimens with IgG4-RSD aortitis frequently exhibit a nongranulomatous lymphocytic aortitis with increased numbers of plasma cells (Figure 5, G). Eosinophils and obliterative phlebitis are also frequently observed. ${ }^{16}$ Storiform fibrosis, when present, is strongly suggestive of the diagnosis, although precise diagnostic criteria are lacking.

Approximately $5 \%$ to $20 \%$ of cases of thoracic aortitis may be related to IgG4-RSD. ${ }^{27-29}$ Although this is a significant number, it is much less so than the $50 \%$ of cases of abdominal aortitis believed to be associated with IgG4-RSD. ${ }^{30}$ Complicating the issue, there is a lack of true consensus regarding diagnostic criteria for this entity. Many studies lack adequate serologic correlation and long-term follow-up, leading some authors to contend that elevated tissue IgG4 levels may not be specific to this diagnosis. ${ }^{31}$

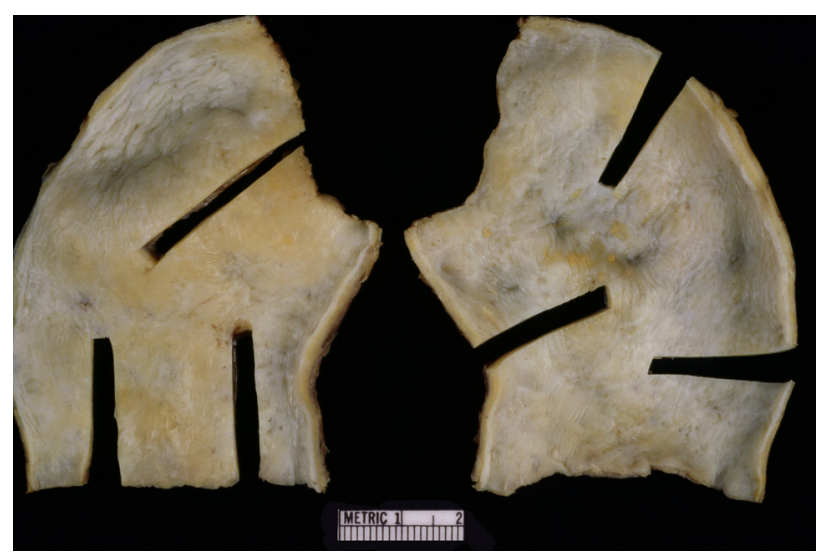

FIGURE 3. Dissection and sampling of ascending aortic specimens. The specimen is measured, opened, and sampled. The intimal wrinkling in this example is a clue to the underlying aortitis.

\section{Infectious Aortitis}

Infectious aortitis is generally categorized as either pyogenic or syphilitic, the latter of which has become vanishingly rare in the developed world. Before the widespread use of antibiotics, cardiovascular complications were a significant cause of morbidity and mortality and patients with tertiary syphilis. The diagnosis of either may represent a complex clinical picture with a relatively high complication rate, necessitating an interdisciplinary team for treatment.

Syphilitic aortitis. Syphilitic aortitis has a strong predilection toward involvement of the ascending aorta. The aneurysms are often remarkably dilated, often with concomitant aortic regurgitation. Intimal wrinkling, similar to that seen in noninfectious aortitis, is also commonplace. Histopathologically, lymphoplasmacytic infiltrates predominate in the media and adventitia, sometimes with an obliterative endarteritis of the vasa vasora. Rarely, demonstration of the offending microorganism, Treponema pallidum, can be achieved with use of a silver-impregnated stain (eg, Warthin-Starry).

Pyogenic aortitis. Pyogenic aortitis uncommonly affects the ascending aorta. Bacteria may involve the aorta by direct implantation on the intimal surface (eg, septicemia or endocarditis), by direct extension from an extravascular site, by traumatic inoculation, or by embolization to the vasa vasora. Placement of aortic grafts (either open or endovascular) may also serve as an entryway for microorganisms. ${ }^{32}$ Staphylococcus aureus, Streptococcus species, Salmonella species, gram-negative rods, and fungi have been reported in infectious aneurysms; however the infrequent nature of ascending aortic involvement precludes a assessment of site-specific etiologic trends. ${ }^{33,34}$

Histopathologically, pyogenic aortitis is characterized by a neutrophilic infiltrate (Figure 5, $H$ ), usually with profound injury to the underlying vessel. Microorganisms may be identified with the use of special stains; however, intraoperative culture is typically of higher yield (particularly if antibiotic therapy has not yet been initiated). 


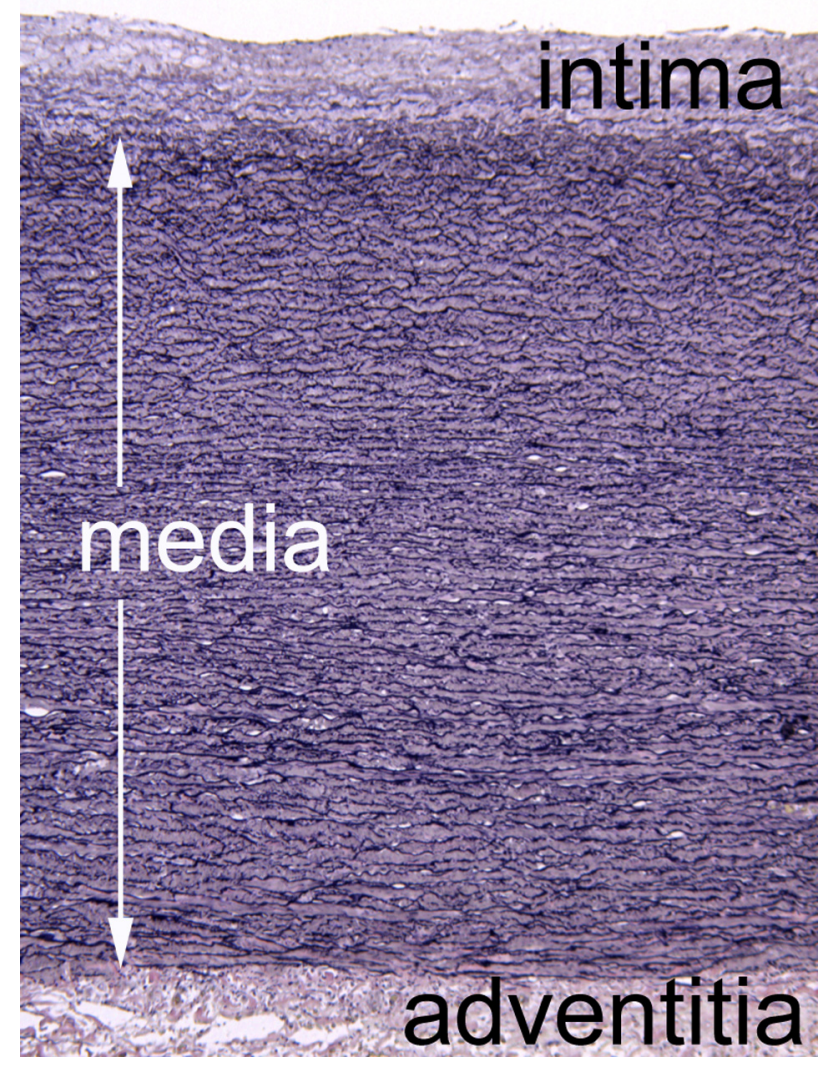

FIGURE 4. Normal microanatomy of the aorta on elastic stain. This photomicrograph exhibits a normal, full-thickness section of aortic wall. The elastic lamellae are parallel and even in distribution from the adventitia to the intima (Verhoeff-van Gieson staining).

\section{COMPLICATIONS}

\section{Aneurysm}

Aneurysm is broadly defined as an expansion of a structure beyond its normal expected borders. Development of aneurysm is commonplace in the setting of inflammatory aortic disease and is the primary driver for surgical resection in the setting of aortitis. The progression of aortic aneurysms depends on a complex set of interactions between the constituents of the aortic wall, including smooth muscle myocytes, macrophages, endothelial cells, and fibroblasts. ${ }^{35}$
All types of inflammatory aortic disease may produce aneurysm, although to varying degrees. Atherosclerotic aneurysms, for example, of the ascending aorta are quite infrequent. $^{36}$ Although TA is often characterized as prototypically producing stenotic lesions, more than $50 \%$ of individuals with TA may also show development of aneurysms. ${ }^{37}$

Infectious aortitis may also result in aneurysm, with syphilitic aortitis producing massive ascending aortic aneurysms that may erode into adjacent structures with or without rupture or dissection. ${ }^{38}$ Syphilitic aortitis invariably affects the ascending aorta, usually sparing the aortic sinuses. ${ }^{39}$ Nonsyphilitic infectious aortitis may also produce aneurysms that, in contrast to noninfectious aortitis, are often saccular (or noncircumferential) and multiple. ${ }^{32}$ Involvement of the ascending aorta by pyogenic aortitis is unusual. Rapid progression of aneurysm size may ensue with pyogenic aortitis, and appropriate microorganism identification and antibiotic therapy are paramount. ${ }^{32,33}$

Approximately $5 \%$ of patients undergoing surgical resection of an aortic aneurysm are found to have underlying aortitis. $^{40}$ Individuals with biopsy-proven GCA involving the temporal or occipital arteries, for example, have been shown to be 17 times more likely to have development of thoracic aortic aneurysms than a healthy age- and sex-matched population. ${ }^{41}$ With that said, many patients with a diagnosis of aortitis, most of whom are older men, do not have manifestations of a systemic vasculitis; the term isolated aortitis has been applied in this situation. ${ }^{15}$ Few patients with such will go on to have development of autoimmune symptoms, aneurysms, or additional manifestations of systemic vasculitis, calling into question whether anti-inflammatory treatment is indicated. ${ }^{10,15,42}$

\section{Dissection and Rupture}

Aortic dissection is the result of an in intimal tear that allows entry of blood into the media, splaying apart the elastic lamella. If the dissection track extends through the media, without breaching the adventitia, a contained rupture (socalled false aneurysm) is formed. Extension through the adventitia is a frank rupture. Aortic dissection or rupture may occur in an aneurysmal or nonaneurysmal segment. ${ }^{11}$

FIGURE 5. Disease histologic characteristics. A, Atherosclerosis with an intense chronic inflammatory infiltrate (hematoxylin and eosin staining). B, Giant cell arteritis involving the aorta with numerous giant cells and associated chronic inflammatory infiltrate. Note the puckered appearance of the intimal surface (arrows), the histologic correlate to gross intimal wrinkling ("tree barking"; hematoxylin and eosin staining). C, A Verhoeff-van Gieson elastic stain highlights the "moth-eaten" appearance of the aortic media, caused by underlying giant cell arteritis involving the aorta. D, Laminar medial necrosis, although not specific for aortitis, may occasionally be seen in areas away from active inflammation, characterized by smooth muscle myocyte nuclear loss and collapsed or condensed elastic lamellae (arrowheads; Verhoeff-van Gieson staining). E, Granulomatosis with polyangiitis, characterized by irregular basophilic (purple-blue) necrosis throughout the aortic wall, so-called geographic necrosis (hematoxylin and eosin staining). F, Sarcoidosis periaortitis, with numerous well-formed nonnecrotizing granulomas in the adventitia (asterisks) and focal extension into the media (hematoxylin and eosin staining). G, Purulent aortitis, characterized by exuberant neutrophilic infiltrate throughout the involved aorta (hematoxylin and eosin staining). H, Pyogenic aortitis, characterized by a neutrophilic infiltrate with tissue destruction. 

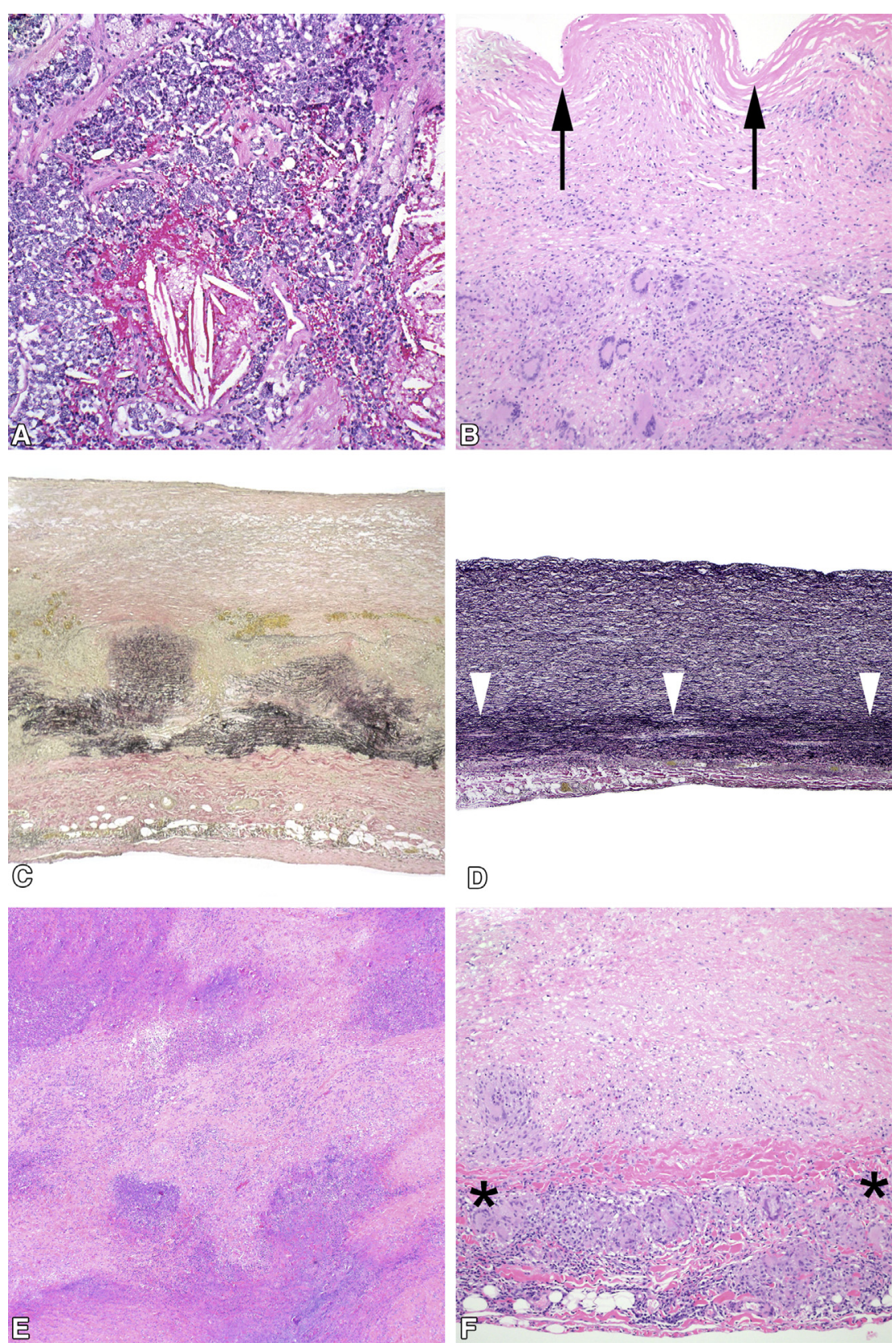

(D)
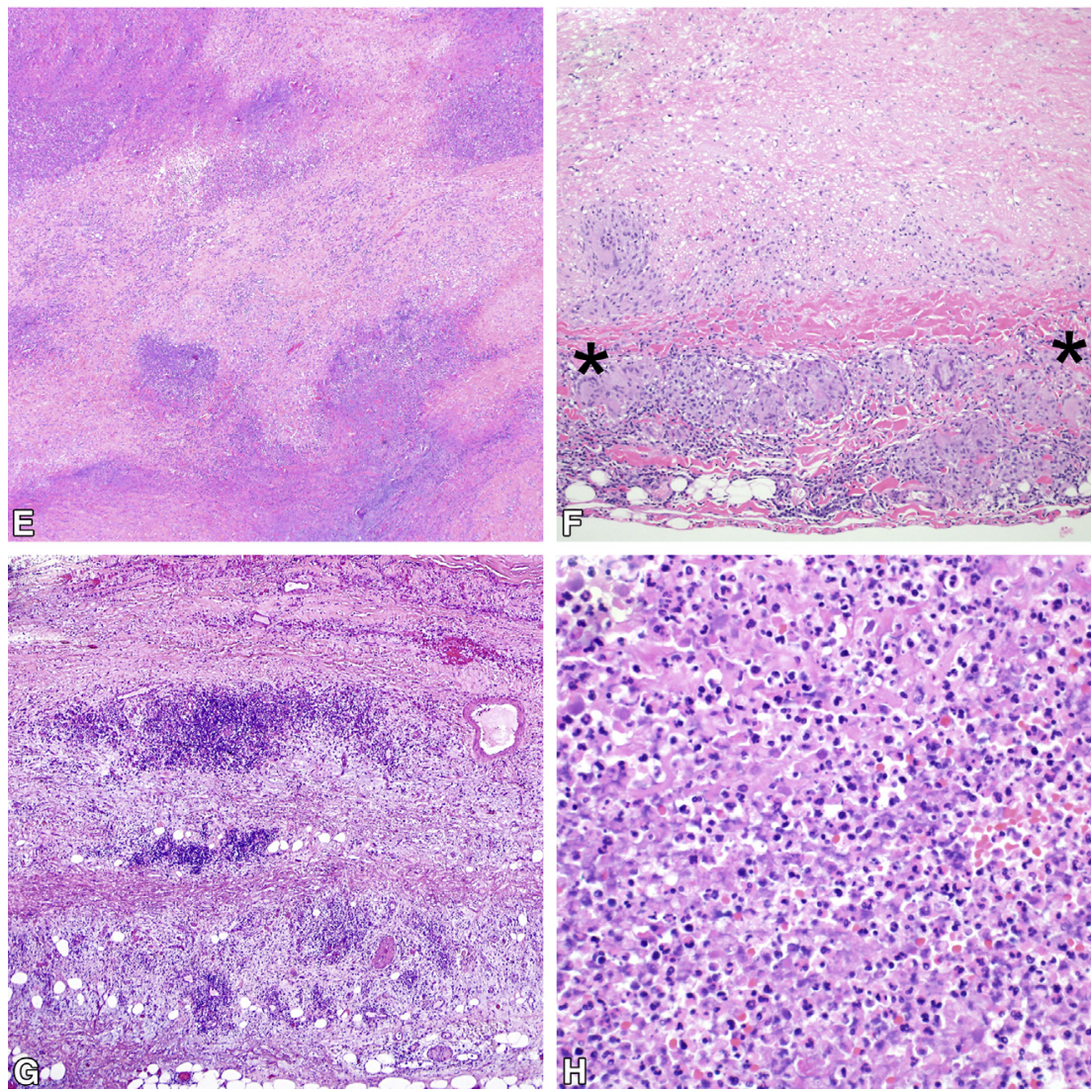
In a population-based cohort study, Evans and colleagues ${ }^{41}$ described 4 individuals in a cohort of 94 patients with biopsy-proven GCA (4\%) as having aortic dissection develop. There is relatively low rate of rupture observed in individuals with TA, probably because of the dense fibrotic thickening of the aortic wall that is common in the late phase of the disease. GPA has also been reported to cause dissection. ${ }^{20}$

Infectious aortitis may also result in dissection or rupture. ${ }^{43}$ In cases where an infectious etiology is suspected, rapid surgical intervention and débridement may lower the risk of dissection or rupture. ${ }^{44}$ Imaging studies demonstrating periaortic thickening and tissue gas collections can provide clues to the underlying diagnosis and may suggest impending rupture. ${ }^{45,46}$

\section{CONCLUSIONS}

Inflammatory aortic diseases that affect the ascending aorta are heterogeneous but are similar in many aspects. Both gross and histologic features can be suggestive of an underlying diagnosis, but correlation with clinical, radiologic, and serologic information is often essential. Although the Chapel Hill consensus has provided an excellent framework though the years, its histopathologic application is imperfect. ${ }^{47}$ Some authors have suggested using schemes that separate into necrotizing versus non-necrotizing aortitis, but this classification may be too broad for clinical purposes. $^{42}$

In an attempt to address these issues, The Society for Cardiovascular Pathology is preparing a consensus statement on the nomenclature of inflammatory aortic disease. The classification will be broadly applicable to practicing pathologists and will allow the appropriate correlation with clinical parameters to form a consensus diagnosis. Continued multidisciplinary collaboration among surgeons, clinicians, and pathologists will be of utmost importance in refining diagnostic categories and ultimately improving patient outcomes.

\section{References}

1. Faury G. Function-structure relationship of elastic arteries in evolution: from microfibrils to elastin and elastic fibres. Pathol Biol. 2001;49:310-25.

2. Wolinsky H, Glagov S. A lamellar unit of aortic medial structure and function in mammals. Circ Res. 1967;20:99-111.

3. Wolinsky H, Glagov S. Comparison of abdominal and thoracic aortic medial structure in mammals. Deviation of man from the usual pattern. Circ Res. $1969 ; 25: 677-86$

4. Kritharis EP, Iliopoulos DC, Papadodima SA, Sokolis DP. Effects of aneurysm on the mechanical properties and histologic structure of aortic sinuses. Ann Thorac Surg. 2014;98:72-9.

5. Cheung C, Bernardo AS, Trotter MW, Pedersen RA, Sinha S. Generation of human vascular smooth muscle subtypes provides insight into embryological origin-dependent disease susceptibility. Nat Biotechnol. 2012;30:165-73.

6. Stone JR, Basso C, Baandrup UT, Bruneval P, Butany J, Gallagher PJ, et al. Recommendations for processing cardiovascular surgical pathology specimens: a consensus statement from the Standards and Definitions Committee of the Society for Cardiovascular Pathology and the Association for European Cardiovascular Pathology. Cardiovasc Pathol. 2012;21:2-16.
7. VanderLaan PA, Reardon CA, Getz GS. Site specificity of atherosclerosis: siteselective responses to atherosclerotic modulators. Arterioscler Thromb Vasc Biol. 2004;24:12-22.

8. McLenachan JM, Vita J, Fish DR, Treasure CB, Cox DA, Ganz P, et al. Early evidence of endothelial vasodilator dysfunction at coronary branch points. Circulation. 1990;82:1169-73.

9. Hellmann DB, Grand DJ, Freischlag JA. Inflammatory abdominal aortic aneurysm. JAMA. 2007;297:395-400.

10. Miller DV, Isotalo PA, Weyand CM, Edwards WD, Aubry MC, Tazelaar HD. Surgical pathology of noninfectious ascending aortitis: a study of 45 cases with emphasis on an isolated variant. Am J Surg Pathol. 2006;30:1150-8.

11. Nuenninghoff DM, Hunder GG, Christianson TJ, McClelland RL, Matteson EL. Incidence and predictors of large-artery complication (aortic aneurysm, aortic dissection, and/or large-artery stenosis) in patients with giant cell arteritis: a population-based study over 50 years. Arthritis Rheum. 2003;48:3522-31.

12. Bossert M, Prati C, Balblanc JC, Lohse A, Wendling D. Aortic involvement in giant cell arteritis: current data. Joint Bone Spine. 2011;78:246-51.

13. Daumas A, Rossi P, Bernard-Guervilly F, Frances Y, Berbis J, Durand JM, et al. [Clinical, laboratory, radiological features, and outcome in 26 patients with aortic involvement amongst a case series of 63 patients with giant cell arteritis]. Rev Med Interne. 2014;35:4-15. French.

14. Homme JL, Aubry MC, Edwards WD, Bagniewski SM, Shane Pankratz V, Kral CA, et al. Surgical pathology of the ascending aorta: a clinicopathologic study of 513 cases. Am J Surg Pathol. 2006;30:1159-68.

15. Liang KP, Chowdhary VR, Michet CJ, Miller DV, Sundt TM, Connolly HM, et al. Noninfectious ascending aortitis: a case series of 64 patients. J Rheumatol. 2009; 36:2290-7.

16. Miller DV, Maleszewski JJ. The pathology of large-vessel vasculitides. Clin Exp Rheumatol. 2011;29(1 Suppl 64):S92-8.

17. Lane SE, Watts R, Scott DG. Epidemiology of systemic vasculitis. Curr Rheumatol Rep. 2005; 7:270-5.

18. Lutalo PM, D'Cruz DP. Diagnosis and classification of granulomatosis with polyangiitis (aka Wegener's granulomatosis). J Autoimmun. 2014;48-9: 94-8.

19. Carels T, Verbeken E, Blockmans D. p-ANCA-associated periaortitis with histological proof of Wegener's granulomatosis: case report. Clin Rheumatol. 2005; 24:83-6.

20. Blockmans D, Baeyens H, Van Loon R, Lauwers G, Bobbaers H. Periaortitis and aortic dissection due to Wegener's granulomatosis. Clin Rheumatol. 2000;19:161-4.

21. Thomas KW, Hunninghake GW. Sarcoidosis. JAMA. 2003;289:3300-3.

22. Hou L, Masuda M, Akashi M, Kido S, Takase Y, Kai K, et al. Metachronous aortic aneurysms due to sarcoidosis. Pathol Int. 2012;62:335-8.

23. Maeda S, Murao S, Sugiyama T, Utaka I, Okamoto R. Generalized sarcoidosis with "sarcoid aortitis" Acta Pathol Jpn. 1983;33:183-8.

24. Ishii A, Hoshii Y, Nakashima T, Umemoto S, Nakamura H, Tanaka N, et al. Sarcoidosis with pulmonary hypertension exacerbated by Takayasu-like large vessel vasculitis. Pathol Int. 2011;61:546-50.

25. Weiler V, Redtenbacher S, Bancher C, Fischer MB, Smolen JS. Concurrence of sarcoidosis and aortitis: case report and review of the literature. Ann Rheum Dis. 2000;59:850-3.

26. Khosroshahi A, Carruthers MN, Deshpande V, Unizony S, Bloch DB, Stone JH. Rituximab for the treatment of IgG4-related disease: lessons from 10 consecutive patients. Medicine (Baltimore). 2012;91:57-66.

27. Stone JH, Khosroshahi A, Deshpande V, Stone JR. IgG4-related systemic disease accounts for a significant proportion of thoracic lymphoplasmacytic aortitis cases. Arthritis Care Research (Hoboken). 2010;62:316-22.

28. Laco J, Steiner I, Holubec T, Dominik J, Holubcova Z, Vojacek J. Isolated thoracic aortitis: clinicopathological and immunohistochemical study of 11 cases. Cardiovasc Pathol. 2011;20:352-60.

29. Koo BS, Koh YW, Hong S, Kim YJ, Kim YG, Lee CK, et al. Frequency of immunoglobulin G4-related aortitis in cases with aortic resection and their clinical characteristics compared to other aortitises. Int J Rheum Dis. 2014;17: 420-4.

30. Kasashima S, Zen Y, Kawashima A, Endo M, Matsumoto Y, Kasashima F. A new clinicopathological entity of IgG4-related inflammatory abdominal aortic aneurysm. J Vasc Surg. 2009;49:1264-71; discussion 1271.

31. Agaimy A, Weyand M, Strecker T. Inflammatory thoracic aortic aneurysm (lymphoplasmacytic thoracic aortitis): a 13-year-experience at a German Heart Center with emphasis on possible role of IgG4. Int J Clin Exp Pathol. 2013;6: 1713-22.

32. Ting AC, Cheng SW, Ho P, Poon JT, Tsu JH. Surgical treatment of infected aneurysms and pseudoaneurysms of the thoracic and abdominal aorta. Am J Surg. 2005;189:150-4. 
33. Carreras M, Larena JA, Tabernero G, Langara E, Pena JM. Evolution of salmonella aortitis towards the formation of abdominal aneurysm. Eur Radiol. 1997;7:54-6.

34. Silva ME, Malogolowkin MH, Hall TR, Sadeghi AM, Krogstad P. Mycotic aneurysm of the thoracic aorta due to Aspergillus terreus: case report and review. Clin Infect Dis. 2000;31:1144-8.

35. Majesky MW, Dong XR, Hoglund VJ. Parsing aortic aneurysms: more surprises. Circ Res. 2011;108:528-30.

36. Isselbacher EM. Thoracic and abdominal aortic aneurysms. Circulation. 2005; 111:816-28.

37. Hotchi M. Pathological studies on Takayasu arteritis. Heart Vessels Suppl. 1992; 7:11-7.

38. Tavora F, Burke A. Review of isolated ascending aortitis: differential diagnosis, including syphilitic, Takayasu's and giant cell aortitis. Pathology. 2006;38:302-8.

39. Roberts WC, Ko JM, Vowels TJ. Natural history of syphilitic aortitis. Am J Cardiol. 2009; 104:1578-87.

40. Rojo-Leyva F, Ratliff NB, Cosgrove DM 3rd, Hoffman GS. Study of 52 patients with idiopathic aortitis from a cohort of 1,204 surgical cases. Arthritis Rheum. 2000;43:901-7.
41. Evans JM, O'Fallon WM, Hunder GG. Increased incidence of aortic aneurysm and dissection in giant cell (temporal) arteritis. A population-based study. Ann Intern Med. 1995;122:502-7.

42. Burke AP, Tavora F, Narula N, Tomaszewski JE, Virmani R. Aortitis and ascending aortic aneurysm: description of 52 cases and proposal of a histologic classification. Hum Pathol. 2008;39:514-26.

43. Park BS, Min HK, Kang do K, Jun HJ, Hwang YH, Jang EJ, et al. Stanford type A aortic dissection secondary to infectious aortitis: a case report. J Korean Med Sci. 2013;28:485-8.

44. Foote EA, Postier RG, Greenfield RA, Bronze MS. Infectious aortitis. Curr Treat Options Cardiovasc Med. 2005;7:89-97.

45. Lopes RJ, Almeida J, Dias PJ, Pinho P, Maciel MJ. Infectious thoracic aortitis: a literature review. Clin Cardiol. 2009;32:488-90.

46. Malouf JF, Chandrasekaran K, Orszulak TA. Mycotic aneurysms of the thoracic aorta: a diagnostic challenge. Am J Med. 2003;115:489-96.

47. Jennette JC, Falk RJ, Bacon PA, Basu N, Cid MC, Ferrario F, et al. 2012 revised International Chapel Hill Consensus Conference Nomenclature of Vasculitides. Arthritis Rheum. 2013;65:1-11. 